\title{
Complete response of giant desmoplastic small round cell tumor treated with chemoradiotherapy: A case report
}

\author{
SHUO ZHANG ${ }^{1,2^{*}}$, YONG ZHANG ${ }^{2 *}$, YONG-HUA YU ${ }^{2}$ and $\mathrm{JIA} \mathrm{LI}^{2}$ \\ ${ }^{1}$ Department of Oncology, Jinan Central Hospital, Shangdong University, Jinan, Shandong 250002; \\ ${ }^{2}$ Radiation Oncology Ward 2, Shandong Cancer Hospital and Institute, Jinan, Shandong 250117, P.R. China
}

Received November 14, 2014; Accepted October 2, 2015

DOI: $10.3892 / \mathrm{ol} .2015 .4024$

\begin{abstract}
Desmoplastic small round cell tumor (DSRCT) is a rare tumor that mainly affects adolescents, and typically involves the abdominal and pelvic peritoneum. The present study reports one case of giant DSRCT, treated with concurrent chemoradiotherapy, and reviews the available medical literature. A 38-year-old man presented with a 3-month history of pain in the left lower abdomen and nausea, associated with decreased appetite and weight loss. Computed tomography (CT) showed a $12.3 \times 7.9 \mathrm{~cm}$ confluent solid mass in the lower abdomen and pelvic cavity. The patient underwent exploratory laparotomy and the final pathological diagnosis was DSRCT. Following laparotomy, the patient was treated with external beam radiotherapy to the whole abdomen and pelvis to a dose of $40 \mathrm{~Gy}$ plus a $20 \mathrm{~Gy}$ boost to the residual disease. The results indicated that synchronous chemotherapy with cyclophosphamide, adriamycin and cisplatin combined with radiotherapy significantly improved locoregional control of DSRCT and a complete response, as measured by CT assessment 2 months subsequent to radiotherapy. In conclusion, DSRCT is a rare malignancy requiring multidisciplinary treatment, including surgery, chemotherapy and radiotherapy. The results of the present study confirm that radiotherapy has a significant role in the treatment of advanced abdominal DSRCT and may contribute to durable remission.
\end{abstract}

\section{Introduction}

Desmoplastic small round cell tumor (DSRCT), a rare, high-grade malignant neoplasm, which mainly affects young men and adolescents, was first described as a mesenchymal entity with distinct clinicopathological features by Gerald and

Correspondence to: Mr. Yong-Hua Yu, Radiation Oncology Ward 2, Shandong Cancer Hospital and Institute, 440 Jiyan Road, Jinan, Shandong 250117, P.R. China

E-mail: sdyonghuayu@163.com

*Contributed equally

Key words: desmoplastic small round cell tumor, chemotherapy, radiotherapy
Rosai in 1989 (1). It has been reported that 95\% of DSRCTs are located in the abdominal cavity and pelvis, while $<5 \%$ of cases may affect other organs (2). Diagnosis is typically based on the histological and immunohistochemical features of the tumor. A specific chromosomal translocation, $t(11 ; 22)$ (p13;q12), has been identified in DSRCT and is increasingly used to confirm diagnosis (3). The prognosis for patients with DSRCT remains very poor, particularly for those with metastatic diseases (3-year survival rate, 29\%; 5-year survival rate, $18 \%$ ) (4). The standard treatment protocol for DSRCT has not been well established.

The present study reports a case in which this uncommon tumor was too large to be completely resected, and therefore concurrent radiotherapy and chemotherapy [consisting of cyclophosphamide, doxorubicin and cisplatin (CAP)] were utilized. This case is reported with the goal of contributing valuable information about this unusual tumor to the current literature.

\section{Case report}

In May 2014, a 38-year-old Chinese man was admitted to Shandong Cancer Hospital and Institute, with a 3-month history of pain in the lower left abdomen, and nausea associated with decreased appetite and weight loss. There was no history of fever or jaundice, and the patient had no relevant personal or family history of malignancy.

On physical examination, abdominal distension with shifting dullness was identified. Ultrasonography of the abdomen revealed multiple solid-cystic mass lesions in the peritoneal cavity and pelvis, and a small number of ascites. Computed tomography (CT) revealed a $12.3 \times 7.9 \mathrm{~cm}$ confluent solid mass, located in the upper and lower abdomen, which demonstrated moderate, heterogeneous enhancement. Furthermore, several other soft-tissue masses were also identified in the pelvic cavity, as well as lymphadenopathy within the retroperitoneum (Fig. 1). In addition, the patient's left renal pelvis and ureter were dilated. Exploratory laparotomy was undertaken, and a 10.0x8.0x7.0 cm giant mass was identified, originating from the greater omentum. Several 20x20 mm nodules were also found in the retroperitoneal region, as well as the peritoneal and pelvic cavities, which was suggestive of multiple metastases. During surgery, involvement of the left iliac vessels and sigmoid colon was identified; therefore no attempt was made to remove any of the tumor. An metastatic 
A

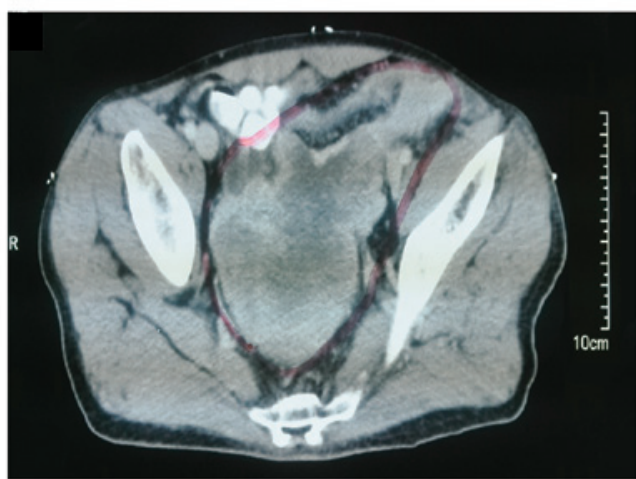

B

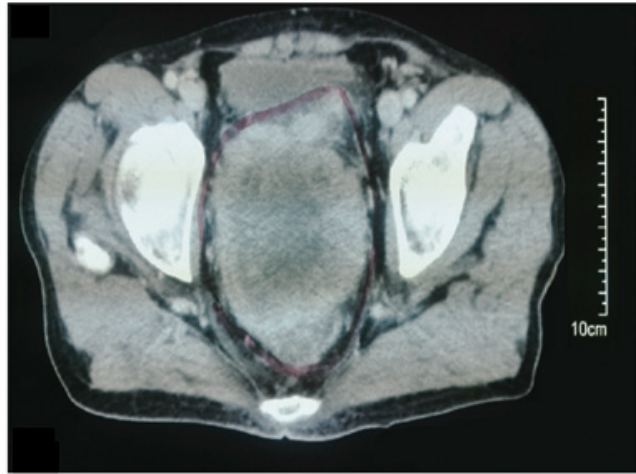

C

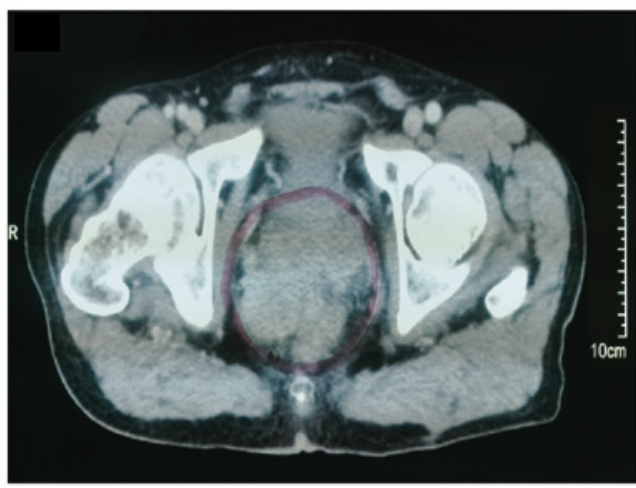

Figure 1. Computed tomography prior to treatment revealed a $12.3 \times 7.9 \mathrm{~cm}$ giant confluent solid mass in the (A) upper and (B) lower abdominal cavity with moderate, heterogeneous enhancement, as well as (C) several additional soft-tissue masses in the pelvic cavity. Red outlines indicate the gross tumor volume.

node resection was undertaken and the resected tissue was subjected to histopathological evaluation.

Histopathological examination of the resected tissue samples revealed that the tumor was malignant, and composed of well-defined nests of small, round blue cells, which were separated by an abundance of desmoplastic stroma (Fig. 2). Immunohistochemistry was as follows: Epithelial membrane antigen $($ EMA $)(+)$, desmin $(\mathrm{DES})(+)$, neuron specific enolase $(\mathrm{NSE})(+)$, synaptophysin[weakly positive $(+/-)]$, calretinin $(+/-)$, cytokeratin $(\mathrm{CK})(-)$, chromogranin-A(-) and cluster of differentiation 99(-). Gene analysis identified the fusion of the Ewing's sarcoma (EWS) and Wilms' tumor (WT1) genes. Based on the aforementioned evidence, a diagnosis of DSRCT was confirmed.

Three-dimensional conformal radiation therapy was administered once per day at 2 Gy/fraction, 5 days/week. The gross tumor volume (GTV) encompassed the entire pelvic cavity, including all gross disease visible on the CT scan used
A

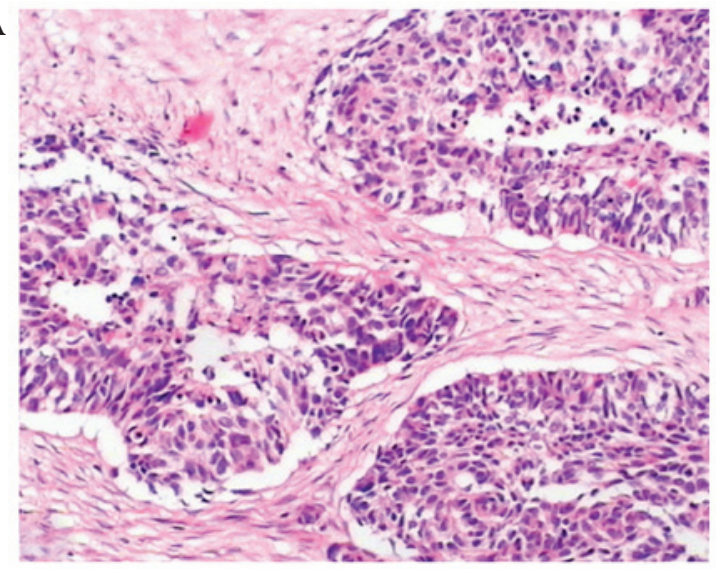

B

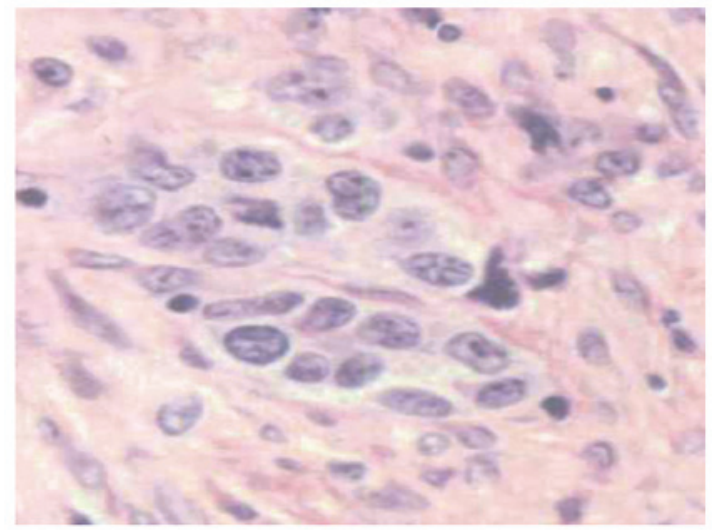

Figure 2. Hematoxylin and eosin staining of a biopsy specimen from the tumor. (A) Nests of small round cells separated by desmoplastic stroma (magnification, x200). (B) Certain cells are arranged in well-defined nests, which are delimited by a cellular desmoplastic stroma (magnification, $\mathrm{x} 400$ ).

for treatment planning. The clinical target volume (CTV) included the GTV $+0.5 \mathrm{~cm}$, and the planning target volume (PTV) encompassed the CTV $+0.5 \mathrm{~cm}$. The CTV and PTV were required to receive $>95 \%$ and $>90 \%$ of the prescribed dose, respectively. The patient was treated with a total of $40 \mathrm{~Gy}$ in 20 fractions. Follow-up CT at a later stage of radiotherapy showed that all of the masses had been significantly reduced in size (Fig. 3). For patients exhibiting a partial response (PR), such as that in the present case, a reduced field boost to $60 \mathrm{~Gy}$ (20 Gy/10 fractions) was implemented.

Concurrent CAP multi-agent systemic chemotherapy regimen, consisting of cyclophosphamide $\left(600 \mathrm{mg} / \mathrm{m}^{2}\right.$, day 1$)$, adriamycin $\left(40 \mathrm{mg} / \mathrm{m}^{2}\right.$, day 1$)$ and cisplatin $\left(25 \mathrm{mg} / \mathrm{m}^{2}\right.$, days 1-3), was administered for three cycles.

The Radiation Therapy Oncology Group/European Organization for Research and Treatment of Cancer acute radiation morbidity scoring system for gastrointestinal (GI) and genitourinary (GU) toxicity grading was used (5). The patient exhibited grade 2 GI and grade 1 GU toxicity scores. Following supportive care, the patient's symptoms rapidly alleviated.

Post-treatment 2-month follow-up evaluation indicated a complete response (CR), as measured by CT assessment (Fig. 4). The patient subsequently succumbed to significant complications induced by recurrence and metastases 30 months following treatment.

Written informed consent for the publication of this report was obtained from the patient. 
A

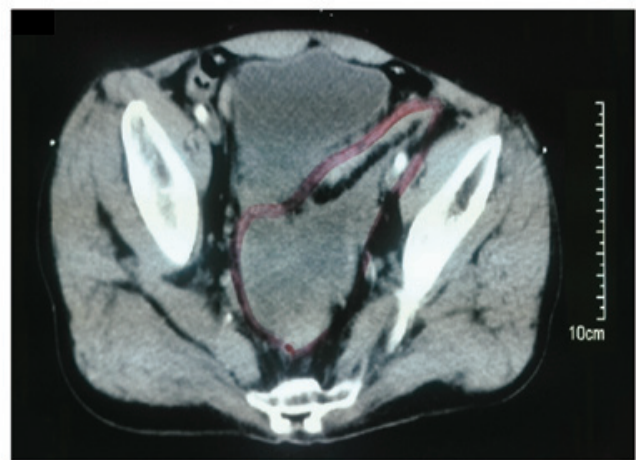

B

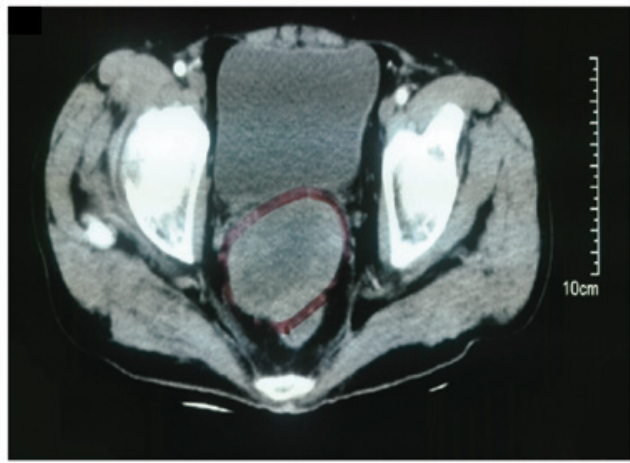

C

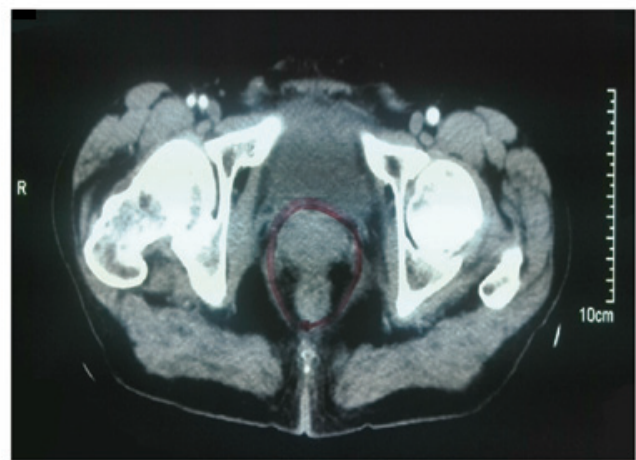

Figure 3. Computed tomography following one month of radiotherapy indicated marked shrinkage of the (A) upper abdominal, (B) lower abdominal and $(\mathrm{C})$ pelvic tumors. Red outlines indicate the gross tumor volume.

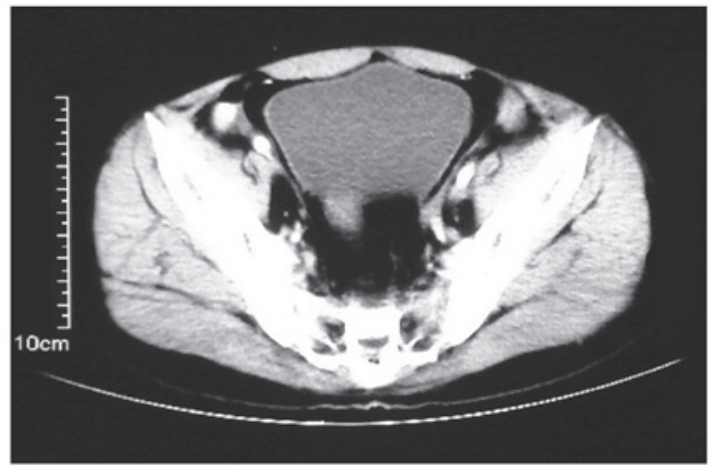

Figure 4. Computed tomography demonstrated complete response of the tumor at 2 months following radiotherapy.

\section{Discussion}

DSRCT is a rare and highly aggressive malignant tumor, and the clinical symptoms of DSRCT are nonspecific and complex. Clinically, DSRCT is common amongst young men, with a mean age at diagnosis of $\sim 22$ years and a $4: 1$ ratio of men to women (6). The most common site of DSRCT occurrence is the pelvis (62\%), followed by wide spreading across the peritoneal surface, and occasionally in certain organs, including the liver, kidneys, ovaries and brain (7). Abdominal pain (52.1\%) and increased abdominal girth $(8.4 \%)$ are the predominant initial symptoms of DSRCT (7). DSRCTs have a tendency towards peritoneal and omental spread, as well as hematogenous metastasis, with easy metastasis to the lung, liver and bone (8).

Clinically, patients may remain asymptomatic for long periods of time and therefore tend to be diagnosed at a relatively advanced stage (9). The imaging examination modalities for DSRCT include ultrasound, CT scan, magnetic resonance imaging and fludeoxyglucose-positron emission tomography/CT imaging. CT scan, the most widely used diagnostic technique, identifies multiple, bulky, lobulated, heterogeneous, and peritoneal soft-tissue masses with a predilection for intraperitoneal spread without obvious primary organ involvement, and may identify widespread implant of the tumor (10). As demonstrated in the present study, abdominopelvic DSRCT may develop into bulky and multiple masses that displace the neighboring organs. However, radiological examination of DSRCT is also nonspecific, and may simply provide useful information regarding the tumor site, size and efficacy evaluation. Diagnosis is mainly based on pathology, immunohistochemistry (IHC) and cytogenetic analysis. Histologically, DRSCT mainly consists of small round blue cells arranged in nests, separated by an abundant desmoplastic stroma (11). In addition, the neoplastic cells, which typically express epithelial (CK and EMA), mesenchymal (vimentin), myogenic (DES) and neural markers (NSE) in IHC analysis, provide further evidence for confirmative and differential diagnosis (12). Furthermore, DSRCT is associated with a unique chromosomal translocation $\mathrm{t}(11 ; 22)(\mathrm{p} 13 ; \mathrm{q} 13)$, resulting in EWS and WTI gene fusion, which typically occurs in the embryonic mesenchymal cells of the serosa of the abdominal organs (13).

Since this type of tumor is rare, the treatment of DSRCT remains clinically challenging and lacks a standard treatment strategy. Potential therapeutic strategies include, debulking surgery, radiotherapy and chemotherapy. However, which of these techniques exhibits the greatest efficacy remains to be elucidated. Due to the extensive involvement typical of this disease at the time of treatment, complete resection without sacrifice of adjacent organs is usually impossible. However, it is suggested that an attempt should be made to remove as much macroscopic tumor as possible. Primary complete or partial removal of macroscopic disease was only possible in $60 \%$ of patients in a previous study, but was associated with an extended median survival (14). The effect of complete resection of diffused tumors on survival remains to be elucidated due to the infrequency that complete resection may be achieved during surgery.

To date, there is no standard chemotherapeutic regimen for the treatment of DSRCT. Similarly to other small round-cell tumors, DSRCT is alkylator-sensitive and dose-responsive (15). Kushner et al (16) reported a 100\% response rate following treatment of 10 patients with DSRCT with a chemotherapy regimen (P6 protocol) comprised of vincristine, doxorubicin, cyclophosphamide, ifosfamide and etoposide. Zhang et al (9) 
also revealed a trend for improved survival in patients treated with chemotherapy. The median survival of patients treated with chemotherapy was 20 months, which was markedly higher than that of patients not treated with chemotherapy. DSRCT remains too rare to establish chemotherapy guidelines according to published medical literature and the results of the present study.

Radiotherapy, and whole abdominal-pelvic radiotherapy in particular in DSRCT, has not been used extensively due to its acute toxicity and low response rate (17). However, a number of researchers have suggested that whole abdominal-pelvic radiotherapy has certain positive effects, including shrinking tumors and relieving symptoms. Goodman et al (18) reported the case of 21 patients with DSRCT treated with radiotherapy. Following maximal surgical debulking, patients were administered a dose of 30 Gy external beam radiotherapy to the whole abdomen and pelvis. The median follow-up period was 28 months, and the overall survival and relapse-free survival rate 3 years post-treatment was 48 and 19\%, respectively. Median survival was 32 months, while the median time to relapse was 19 months. The majority of relapses were intraperitoneal and/or hepatic. In the present study, pelvic radiotherapy was administered, and resulted in a favorable response by shrinking the local tumor. Radiotherapy resulted in a CR of the disease 2 months subsequent to treatment. Therefore, radiotherapy was suggested to be an effective treatment strategy for DSRCT, as the tumor exhibits multicentric growth in the abdominopelvic cavity and due to the rapid development of accurate radiotherapeutic technology (19). To the best of our knowledge, the present case is the first case of a patient with DSRCT treated with 40 Gy to the initial tumor mass, plus a 20 Gy boost to the residual disease, documented in the literature. Patients treated with radiotherapy often experience significant acute toxicity; however, in the present case, the GU and GI side effects were managed with aggressive medical support and the patient was able to complete the treatment course.

Despite multiple treatment strategies including high-dose chemotherapy regimens active for DSRCT, aggressive debulking surgery, whole abdominal radiation or even autologous stem cell transplant, the prognosis of DSRCT remains poor; the median progression-free survival is 2.6 years, and the progression-free survival 5 years following diagnosis is $18 \%$ (20). Ordóñez (21) indicated that $71 \%(25 / 35)$ of patients succumbed within 8-50 months (mean, 25.2 months). A further study revealed that certain targeted therapeutic agents may be used to treat such lesions, which may suggest further prospective research and indicate that it is reasonable to initiate patient enrollment in clinical trials (22).

To date, the optimal management of DSRCT remains challenging and current schemes lack a significant cure rate despite the use of aggressive treatments (15). Chemoradiotherapy is significant in the treatment of advanced abdominal DSRCT. As a therapeutic measure chemoradiotherapy may relieve symptoms and improve patient outcome. The effectiveness and safety of chemoradiotherapy urgently require improvement prior to use in the treatment of this disease, particularly when patients are unable to tolerate aggressive surgery or the tumors cannot be surgically resected. In addition, earlier diagnosis is critical to the management of patients with DSRCT. Further investigations are required in order to define the optimal therapeutic strategy for this disease.

\section{References}

1. Gerald WL and Rosai J: Case 2. Desmoplastic small cell tumor with divergent differentiation. Pediatr Pathol 9: 177-183, 1989.

2. Bismar TA, Basturk O, Gerald WL, Schwarz K and Adsay NV: Desmoplastic small cell tumor in the pancreas. Am J Surg Pathol 28: 808-812, 2004.

3. Zhang GM, Zhu Y, Gan HL and Ye DW: Testicular desmoplastic small round cell tumor: A case report and review of literature. World J Surg Oncol 12: 227, 2014.

4. Thandassery RB, Elbedawi M, El-Malik H, John AK, Al-Bozom IA and Al Kaabi SR: Intra-abdominal desmoplastic small round cell tumor. Ann Gastroenterol 27: 287-288, 2014

5. Cox JD, Stetz J and Pajak TF: Toxicity criteria of the Radiation Therapy Oncology Group (RTOG) and the European Organization for Research and Treatment of Cancer (EORTC). Int J Radiat Oncol Biol Phys 31: 1341-13476, 1995.

6. Dufresne A, Cassier P, Couraud L, Marec-Bérard P, Meeus P, Alberti L and Blay JY: Desmoplastic small round cell tumor: Current management and recent findings. Sarcoma 2012: 714986, 2012

7. Gil A, Gomez Portilla A, Brun EA and Sugarbaker PH: Clinical perspective on desmoplastic small round-cell tumor. Oncology 67: 231-242, 2004.

8. Perna MJ and Streck CJ: A large solitary desmoplastic small round cell tumor. Am Surg 78: E316-E317, 2012.

9. Zhang J, Xu H, Ren F, Yang Y, Chen B and Zhang F: Analysis of clinicopathological features and prognostic factors of desmoplastic small round cell tumor. Pathol Oncol Res 20: 161-168, 2014.

10. Zhang G, Liu G, Zhao D, Cui X and Li G: Desmoplastic small round cell tumor of the abdomen and pelvis: Clinicopathological characters of 12 cases. ScientificWorld Journal 2014: 549612, 2014.

11. Jordan AH and Pappo A: Management of desmoplastic small round-cell tumors in children and young adults. J Pediatr Hematol Oncol 34 (Suppl 2): S73-S75, 2012.

12. Chang F: Desmoplastic small round cell tumors: Cytologic, histologic, and immunohistochemical features. Arch Pathol Lab Med 130: 728-732, 2006.

13. de Araújo RA and Araújo BJ: Desmoplastic small round cell tumor: Report of 2 cases treated with chemotherapy alone or in combination with bevacizumab. Case Rep Oncol 7: 102-108, 2014.

14. Hassan I, Shyyan R, Donohue JH, Edmonson JH, Gunderson LL, Moir CR, Arndt CA, Nascimento AG and Que FG: Intraabdominal desmoplastic small round cell tumors: A diagnostic and therapeutic challenge. Cancer 104: 1264-1270, 2005.

15. Honoré C, Amroun K, Vilcot L, Mir O, Domont J, Terrier P, Le Cesne A, Le Péchoux C and Bonvalot S: Abdominal desmoplastic small round cell tumor: multimodal treatment combining chemotherapy, surgery, and radiotherapy is the best option. Ann Surg Oncol 22: 1073-1079, 2015.

16. Kushner BH, LaQuaglia MP, Wollner N, Meyers PA, Lindsley KL, Ghavimi F, Merchant TE, Boulad F, Cheung NK, Bonilla MA, et al: Desmoplastic small round-cell tumor: Prolonged progression-free survival with aggressive multimodality therapy. J Clin Oncol 14: 1526-1531, 1996.

17. Desai NB, Stein NF, LaQuaglia MP, Alektiar KM, Kushner BH, Modak S, Magnan HM, Goodman K and Wolden SL: Reduced toxicity with intensity modulated radiation therapy (IMRT) for desmoplastic small round cell tumor (DSRCT): An update on the whole abdominopelvic radiation therapy (WAP-RT) experience. Int J Radiat Oncol Biol Phys 85: e67-e72, 2013.

18. Goodman KA, Wolden SL, La Quaglia MP and Kushner BH: Whole abdominopelvic radiotherapy for desmoplastic small round-cell tumor. Int J Radiat Oncol Biol Phys 54: 170-176, 2002.

19. Yue X, Wang JZ, Tian Y and Wang KJ: Paratesticular desmoplastic small round cell tumor with metastasis: a report of two cases. Kaohsiung J Med Sci 30: 104-105, 2014.

20. Schwarz RE, Gerald WL, Kushner BH, Coit DG, Brennan MF and La Quaglia MP: Desmoplastic small round cell tumors: Prognostic indicators and results of surgical management. Ann Surg Oncol 5: 416-422, 1998.

21. Ordóñez NG: Desmoplastic small round cell tumor: I: A histopathologic study of 39 cases with emphasis on unusual histological patterns. Am J Surg Pathol 22: 1303-1313, 1998.

22. Sankhala KK and Chawla SP: Review: Desmoplastic small round cell tumor: Current treatment approach and role of targeted therapy. Clin Adv Hematol Oncol 7: 476-478, 2009. 